\title{
An appraisal of the application of open-cell foams in automotive SCR systems
}

\author{
Andrea Vespertini ${ }^{1,2, *}$, Augusto Della Torre ${ }^{1, * *}$, Gianluca Montenegro ${ }^{1, * * *}$, \\ Angelo Onorati ${ }^{1, * * *}$, Enrico Tronconi ${ }^{2, * * * * *}$ and Isabella Nova $^{2,{ }^{* * * * * *}}$ \\ ${ }^{1}$ Politecnico di Milano, Department of Energy, Internal Combustion Engines Group, Italy \\ ${ }^{2}$ Politecnico di Milano, Department of Energy, Laboratory of Catalysis and Catalytic Processes, Italy
}

\begin{abstract}
This work aims to investigate the possibility to apply open-cell foams as catalytic substrates in SCR systems for Diesel engines, as a replacement of traditional honeycombs. In the literature, many studies compare the performance of foams and honeycombs as catalytic substrates, showing, in general, a better mass transfer behavior in foams, compensated on the other hand by a higher pressure drop. In this work, we consider the low-pressure injection of Ad-Blue and we evaluate the performance of the open-cell foam in enhancing the mixing and the evaporation of the spray. A Eulerian-Lagrangian CFD model has been adopted to simulate the spray evolution and its interaction with the microstructure of the open-cell foam. The model has been applied to evaluate the spray evaporation and the uniformity of the ammonia distribution in different sections of the substrate. Different operating conditions were tested comparing substrates with different geometrical properties. The results of this preliminary analysis can be regarded as promising, showing the capability of the foam to enhance the mixing of the spray and to achieve a uniform distribution of the ammonia over all the catalyst substrate.
\end{abstract}

\section{INTRODUCTION}

In the past decades, open-cell foams have been largely studied with possible applications in different fields, ranging from the development of heat-exchange devices to the design of compact catalytic reactors, highlighting their interesting performances in terms of high heat/mass-transfer and low pressure drop [1]. With regards to the specific field of the aftertreatment systems for internal combustion engines, several studies compared open-cell foam substrates with traditional honeycombs. In particular, Giani [2] proposed the definition of a performance index to evaluate the mass transfer in relation to the pressure drop induced by the structure: the substrate is more efficient as the mass transfer increases with the same pressure drop or the pressure drop decreases with the same mass transfer. Lucci [3] run a parametric analysis considering different porosities and cell densities of the open-cell foam substrates, demonstrating that open-cell foams, if properly designed (high porosities, low superficial flow velocities), can have higher performance index with respect to traditional honeycombs. Bach [4] reports a vehicle application of an after-treatment system based on open-cell foams mounted on a small heavy-duty truck and tested on the chassis dynamometer, showing promising evidences of a possible application of this technology. In particular, the experimental results showed that, at low exhaust gas mass flow, the emission conversion was comparable with that measured in case of traditional honeycomb, but it was reached with a lower amount of catalyst loading. On the other hand, the test showed that the system with open-cell foams was penalized at high flow rates, requiring a specific optimization to be adopted for this application. However, in the last few years, the recent developments in the Additive Layer Manufacturing (ALM) technology enabled the possibility to design optimized microstructures, giving the spur to further studies in this field of application. In particular, the interest is progressively shifting towards the adoption generic Periodic Open Cell Structures (POCS), which have morphology and properties similar to open-cell foams but are arbitrarily designed with CAD tools and can be manufactured with ALM, allowing for an extreme flexibility in the

\footnotetext{
*Corresponding author: andrea.vespertini@polimi.it

** augusto.dellatorre@polimi.it

*** gianluca.montenegro@polimi.it

${ }^{* * * *}$ angelo.onorati@polimi.it

***** enrico.tronconi@polimi.it

****** isabella.nova@polimi.it
} 
optimization at both the micro-scale and the macro-scale of the device. Recent examples of application in the field of the catalyst substrate design and application to engine ATS are reported in $[5,6]$.

In this scenario, the present work aims to investigate a novel application in the field of after-treatment systems, namely the adoption of an open-cell foam (or POCS) in the Selective Catalytic Reduction (SCR) devices. In this system a crucial key-factor for the overall efficiency of the NOx reduction process is represented by the urea injection and its subsequent mixing with the exhaust gas flow. This process of mixing is traditionally enhanced by the adoption of mixing devices which promote the distribution of the spray droplet uniformly across the catalyst cross section [7]. In this context, the aim of this work is to explore the possibility to design an novel mixing device based on POCS which combines: a) the capability to promote the uniform distribution of the droplets and their mixing with the gas flow; b) the capability to act at the same time as catalytic substrate for the activation of the reactions. To this purpose, a simple cubic cell POCS was considered and a substrate having main dimensions similar to those of a typical SCR was designed. The system was tested under different operating conditions typical of the engine operation, evaluating the performances in terms of enhancement of the evaporation of the liquid droplets and the mixing of the vapour and exhaust gas downstream the surface.

\section{METHODOLOGY}

The aim of this work is to provide a characterization of the behavior of a POCS (Periodic Open Cell Structure) during the injection of water droplets, focusing mainly on its capability to enhance the level of uniformity of the distribution of water vapour / droplets downstream the structure.

The numerical simulation of this benchmark has required the application of a CFD model able to treat the physics of the process, including both the Lagrangian-Eulerian matching and the Spray-Wall interaction, with the possible formation of a thin liquid layer upon the structure. In particular, the model was adopted to test the performances of the POCS using fluiddynamic simulations under different conditions: different gas flow velocity (i.e. different Reynold number) and different temperature of the structure.

To simulate the behavior of the flow through the POCS we applied a model already proposed by [8] based on the correction of the known Navier-Stokes equations of motion with the addition of source terms, which would make it possible to link the Lagrangian (liquid) phase with the Eulerian (fluid). From a physical point of view, the CFD model includes:

- a continuity equation, with the addition of a proper source term including gas/liquid phase transition;

- a momentum equation, which contains a balance between all the forces acting on the control volume, including body forces, surface forces and momenta shared by the droplets;

- $\quad$ an energy equation, considering the whole system thermodynamics for both gas and liquid phases;

- a species equation, which guarantees the balance of species concentration into the control volume through the interaction.

From the literature it is known that the matching condition between Lagrangian and Eulerian phases is quite complicated to treat analytically: this has led to a condition for a minimum fluid-cell dimension, which has to be about 10 times the droplet characteristic diameter, to guarantee a correct description of drag forces, heat exchange and kinematics between spray and fluid. Nevertheless, mesh density had to be also sufficiently high to describe the interaction between the fluid and the walls of the POCS microstructure. Therefore, the optimum computational grid should result from a balance between these two opposite requirements.

Furthermore, in this framework the spray characteristics have been implemented through the use of an object, the parcel, which consistently reduces the computational load: this consists in a statistic ensemble of droplets having the same thermophysical properties, initially fixed by the user, which will evolve during the simulation, representing the whole spectrum of particles in the spray. The solver will receive also the information about the number of parcels injected per second, the injection time law and the diameter distribution of the droplets injected into the system. 


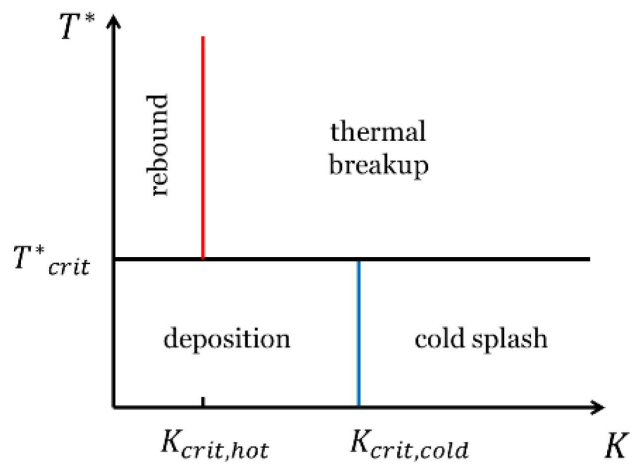

FIGURE 1. Spray-Wall interaction regimes according to the Kuhnke model: in the test cases different regimes are considered by varying the physical parameters.

Then, with regards to the spray-wall interaction description, we adopted Kuhnke's model in order model those phenomena happening between parcels and the POCS. According to the Kuhnke's theory, there are four possible types of interaction, depending on the characteristics of the droplets and the surface. Fig. 1 shows that there are two main parameters which describes the regime of interaction: $T^{*}=\frac{T_{\text {wall }}}{T_{\text {sat }}}$, which is the ratio between POCS temperature and the saturation temperature of water, and $K=W e^{\frac{5}{8}} \times L a^{\frac{1}{8}}$, where We and La are Weber Number and Laplace number, respectively. $T^{*}$ value is set to 1.1. For $\mathrm{K}<\mathrm{K}_{\text {crit,hot }}$ and $T>T^{*}$ a thin layer of vapor is formed around the POCS, preventing it from liquid film formation, leading to a rebound of the impacting parcel, which will keep unchanged its own physical properties. With the increasing of $\mathrm{K}$ beyond $\mathrm{K}_{\text {crithot }}$, liquid film is still not yet formed but each single parcel will be split into fine particles, fulfilling the mass conservation restriction. Considering values of $\mathrm{T}^{*}$ lower than 1.1, parcels can be simply absorbed into the liquid film, for sufficiently low $\mathrm{K}$ values, or can be atomized after having been bound to the liquid film (cold splash regime).

Finally, this model is able to describe the liquid film formation under some reasonable assumption [8]. Summarizing, the film will be descripted by its own physical variables, which will evolve again following Navier-Stokes laws of fluiddynamics; for example, the film will have a velocity which is calculated by balancing different contributions: shear stresses, pressure gradients across the structure, body forces (gravity) and impinging parcels linear momentum transfer. It will be also characterized by its temperature, its water concentration and thickness. Heat exchange between fluid and droplets with the POCS have been correctly evaluated by the model.

\section{CASE STUDY}

\subsection{Geometry}

Our case study mesh consists on a properly designed POCS (Periodic Open Cell Structure), which has been created with dimension such as to approximately fit in an SCR after-treatment system. The base structure (Fig. 2.a) consists of 3 cylindrical struts having diameter $\phi=2 \mathrm{~mm}$ and height $\mathrm{h}=10 \mathrm{~mm}$, intersected with angles of $90^{\circ}$ across the three directions to form a cubic cell. The final substrate has been obtained by a rotation of $90^{\circ}$ with respect to the $y$ - and $\mathrm{z}$ - axes performed over many repeated elementary cells and a cut of the obtained structure, resulting in a POCS of $(84 \times 120 \times 120) \mathrm{mm}$, which stands for around 7 cells on the $x$-axis and 10 on the y-and z- respectively (Fig. 2b). We decided to rotate the geometry in order to maximize the spray-wall interaction which leads to a greater homogenization of the water droplets, resulting in an higher effectiveness with respect to the original structure in intercepting and deviating the particles from their original trajectories. The entire structure has been designed by means of a CAD parametric tools, enabling in perspective the possibility to embed in an optimization workflow. In order to comply with the Lagrangian-Eulerian matching criterion, the surface refinement of the mesh in OpenFOAM has been kept sufficiently low (Fig. 3a): considering that the maximum injected parcels diameter is around $10^{-4} \mathrm{~m}$, the bigger refinement cells are $7.5 \mathrm{~mm}$ while, the fluid inside the POCS can have cells with $1.1 \mathrm{~mm}$ of maximum diameter, which are sufficiently bigger than the droplets characteristic size $\left(\sim 5 \times 10^{-4} / 10^{-5}\right)$. 


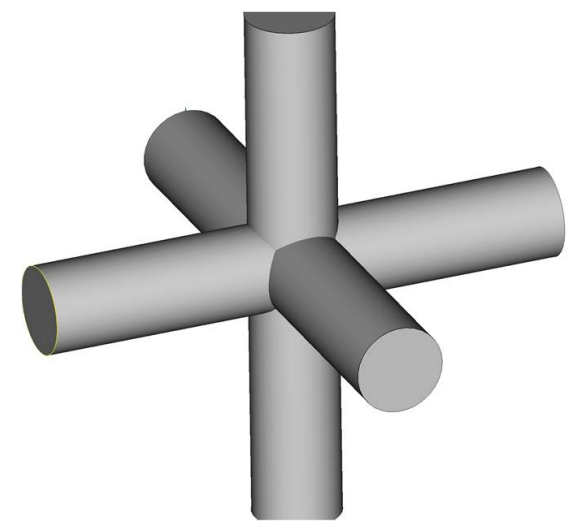

(a)

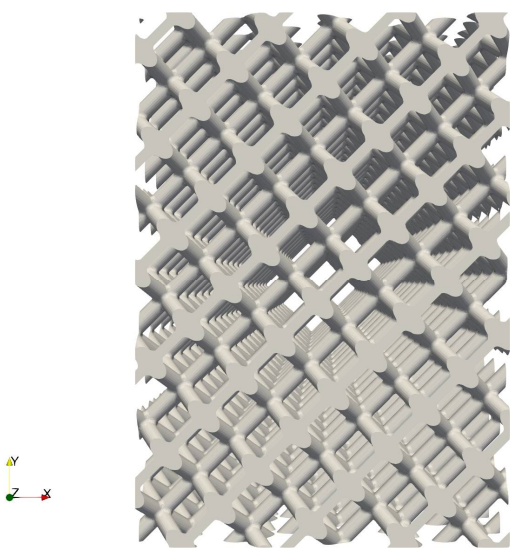

(b)

FIGURE 2. Case study geometry: a) the base structure consists in 3 intersecting cylinders with $2 \mathrm{~mm}$ diameter and $12 \mathrm{~mm}$ of total height; b) in order to maximize spray-wall interaction and homogenization of the injected water, the POCS has been rotated by $90^{\circ}$ with respect to the $\hat{y}$ and $\hat{z}$ axes.

\subsection{Operating conditions and case setup}

The behavior of the POCS was investigated considering a flow of gas, composed mainly by $\mathrm{O}_{2}$ and $\mathrm{N}_{2}$, and characterized by an inlet temperature of $500 \mathrm{~K}$ and velocities in the range $3-10 \mathrm{~m} / \mathrm{s}$. A three holes injector of water is inserted $\sim 3 \mathrm{~mm}$ away from the front of the POCS (Fig. 3b), providing multiple injections, each one lasting 0.2s; the spray is not centered with respect to the face of the POCS, in order to better evaluate homogenization from a more-unbalanced configuration.

As previously mentioned, we tested our POCS for different fluid velocity and temperatures, expecting significant variation of some variables: mass absorbed into liquid film, average $\mathrm{H}_{2} \mathrm{O}$ concentration and mass flow at the outlet, total evaporated mass and homogenization. It is worth noticing that evaporated mass is generated mainly by two factors: evaporation from the liquid film and direct evaporation from the parcels. In typical cases, as it will be shown in the next section, a bigger role is played by the second phenomenon because fluid temperature has been always kept at $500 \mathrm{~K}$ and water saturation temperature is around $373 \mathrm{~K}$; this means that evaporation is favored. Furthermore, once liquid droplets have been injected, they run into a process of progressive atomization, reducing their diameters and, consequently, enhancing evaporation (Fig. 3b).

Parcels contained within the initial spray have a diameter distribution fixed by the user, which is the one depicted in Fig. 4: this curve has a clear peak at around $100 \mu \mathrm{m}$, that corresponds to a drop with a mass of $\sim 5 \times 10^{-10} \mathrm{~kg}$. Parcels have an injection temperature of $293 \mathrm{~K}$.

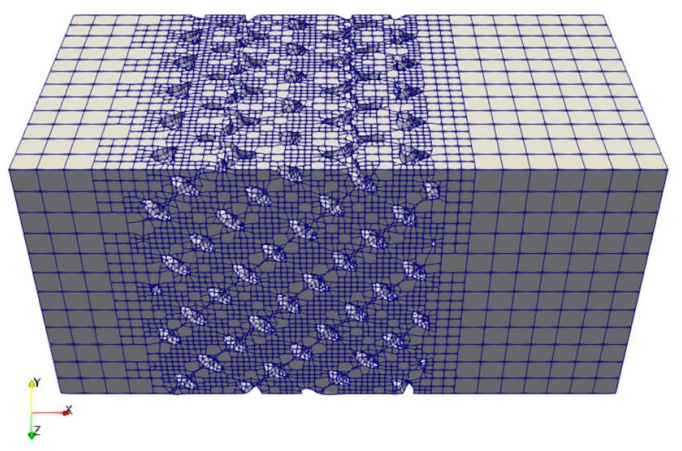

(a)

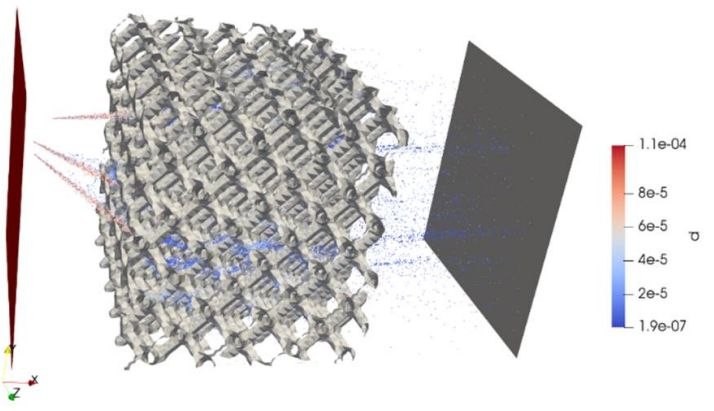

(b)

FIGURE 3. Computational domain: a) the surrounding fluid mesh has a two-times higher level of refinement around the POCS surface, in order to better describe the fluid physics around the microstructure; $b$ ) a three holes injector has been considered, providing four injections of water lasting $0.2 s$ each one. 


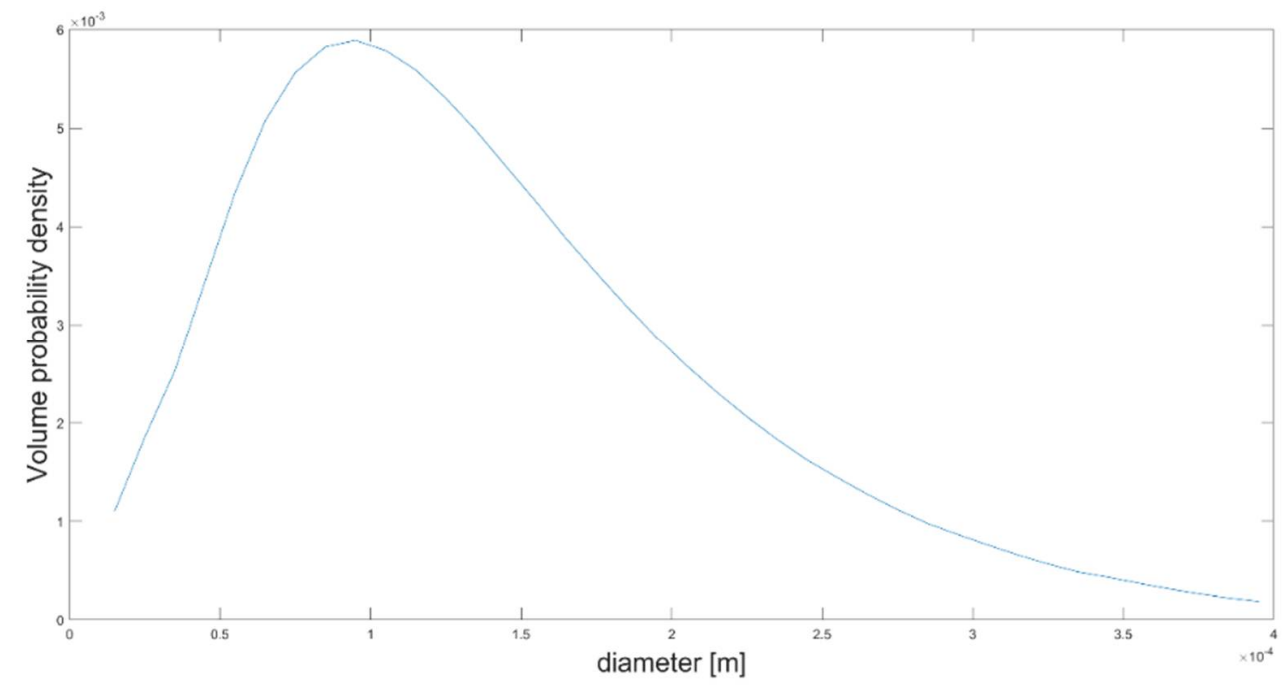

FIGURE 4. Diameter distribution of the injected parcels.

\section{RESULTS AND DISCUSSION}

\subsection{Effects of the gas flow velocity}

In order to characterize the effect of flow regime on the spray mixing and evaporation process, simulations were running for three different gas flow velocities: $3 \mathrm{~m} / \mathrm{s}, 6 \mathrm{~m} / \mathrm{s}$ and $10 \mathrm{~m} / \mathrm{s}$. The temperatures of the incoming gas flow and of the walls of the POCS have been set all to $500 \mathrm{~K}$ in order to be in thermal equilibrium: in this case $T^{*} \cong 1.34>T^{*}{ }_{\text {crit }}$, so we do not expect formation of liquid film around the structure.

In Fig. 5 the average water mass flow at the outlet has been plotted with respect to the time for the different gas flow velocity. In order to compare and to estimate the effectiveness of the system, also the mass flow corresponding to the water injection has been reported in the graph. It can be noticed that at the highest velocity the evaporated mass is slightly less than the $50 \%$ of the injected mass. This percentage decreases as the gas flow velocity is reduced: the enhancement of the evaporation process at high velocity is related to the higher local mixing between droplets and gas induced by the presence of the structure. The gas flow velocity has an effect also on the response time of the system: the maximum vapour flow rate at the outlet is reached faster for high gas velocities due to the higher convection. This effect is particularly appreciable also in the time interval between the subsequent injections.

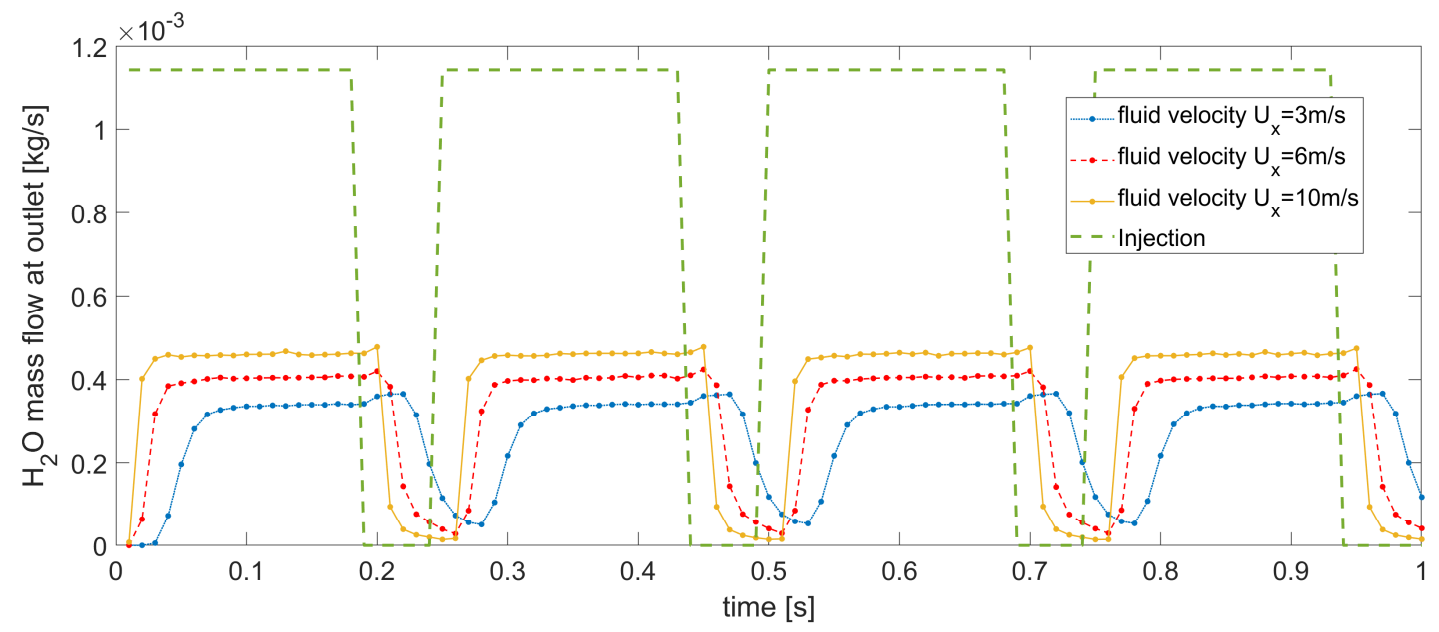

FIGURE 5. Characterization of the POCS behavior under different fluid velocities: mass flow of evaporated water through the outlet patch. 
Fig. 6 reports the evolution over the time of the cumulative mass injected upstrream the POCS and the cumulative water vapour mass leaving the domain at the outlet. Similarly to the previous graph, it can be noticed that the gas flow velocity has a positive effect in promoting the evaporation. Moreover, it should be noticed that for the considered surface temperature of the POCS $(500 \mathrm{~K})$ no liquid film is formed, therefore the water which is not evaporated still consists of liquid droplets which are inside the domain or have left it through the outlet patch.

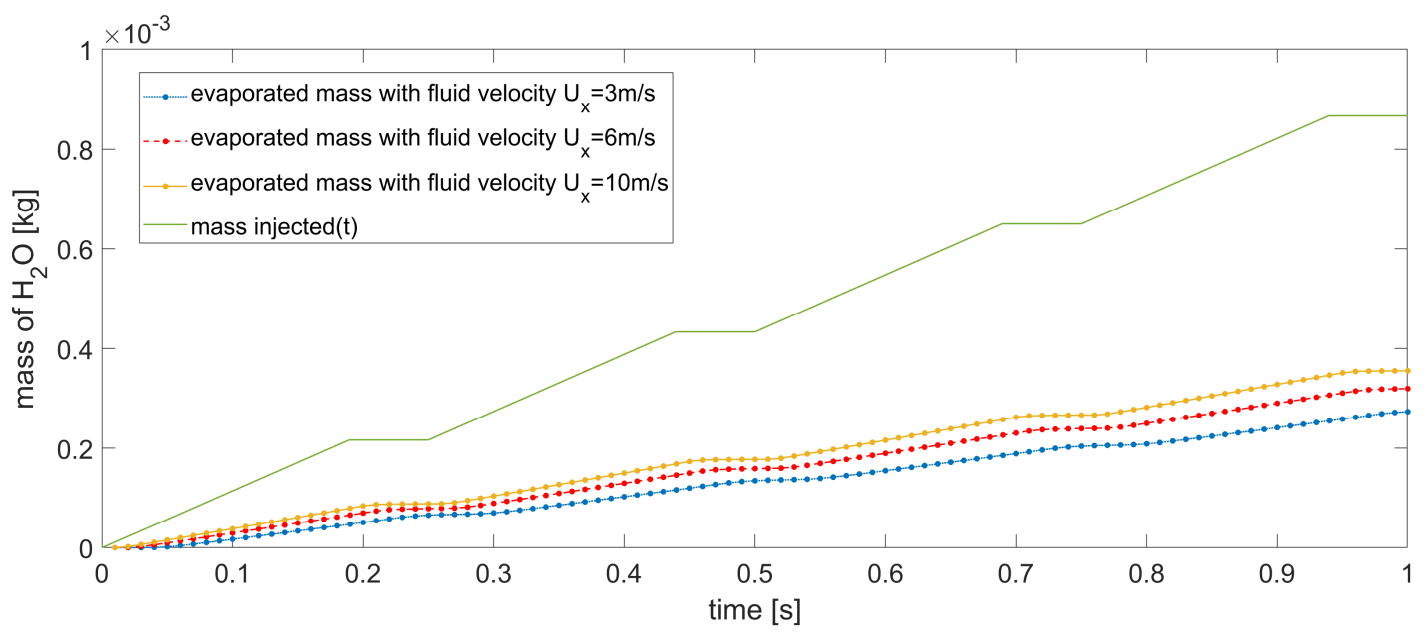

FIGURE 6. Characterization of the POCS behavior under different fluid velocities: injected liquid water mass at the inlet and evaporated water mass at the outlet.

In Fig. 7 the average concentration of water at the outlet is plotted with respect to the time. The average has been performed over the faces of the outlet patch and an estimation of the concentration variability over the patch have been provided through error bars. In particular, the amplitude of the error bars is representative of the range of concentration values which can be found in the $98 \%$ of the faces. As it can be noticed, the average concentration increases as the velocity decreases, inversely with respect to the mass flow: this happens because, as already observed, evaporation increases with the increase of velocity, but the time interval in which we observe the flux is reduced by increasing the velocity.

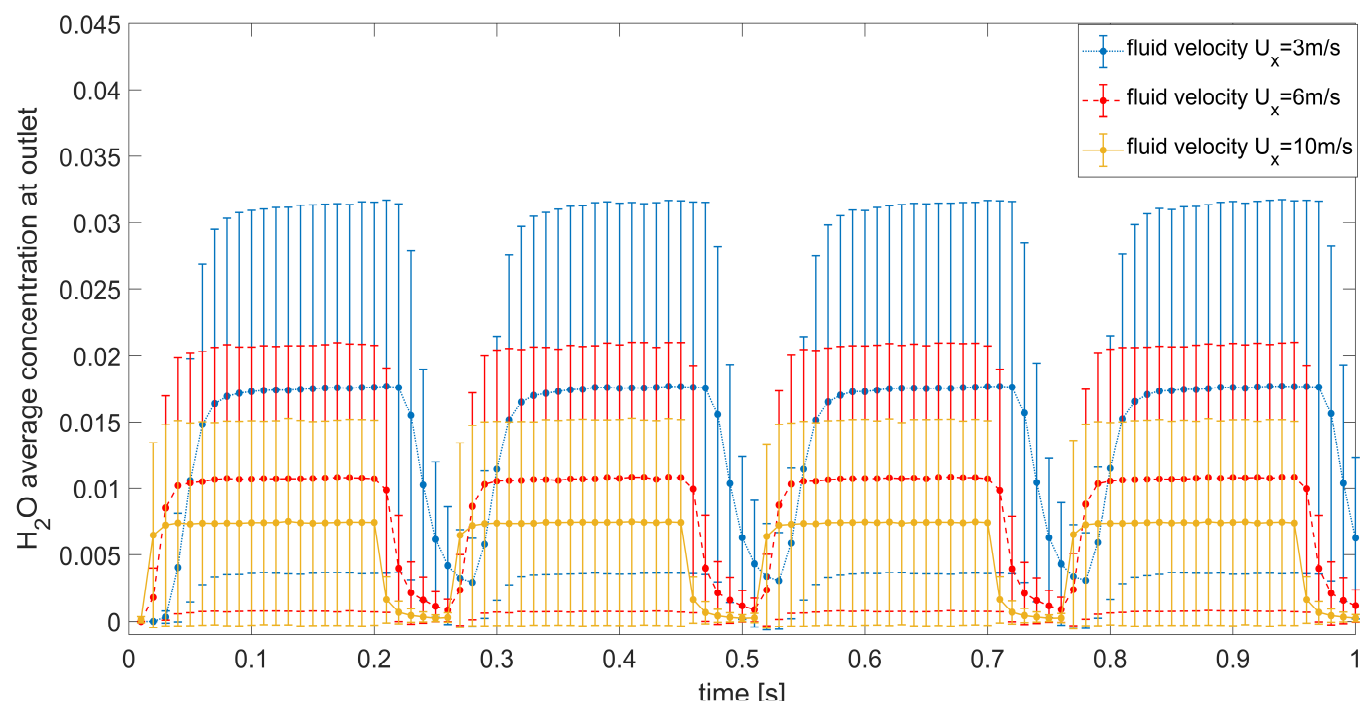

FIGURE 7. Characterization of the POCS behavior under different fluid velocities: vapour concentration at the outlet.

Finally, in Fig. 8 the evolution of the spray across the POCS is shown considering the time $t=0.18 \mathrm{~s}$, corresponding to the final part of the first injection. It can be noticed that the passage through the POCS results in a significant breakup of the injected parcels, which tends to distribute uniformly across the section: this is due to the thermal breakup induced by the 
high temperature of the wall with respect to the saturation temperature of the liquid. Moreover, increasing the gas flow velocity, the downstream parcel diameters reduce as a consequence of the higher evaporation.

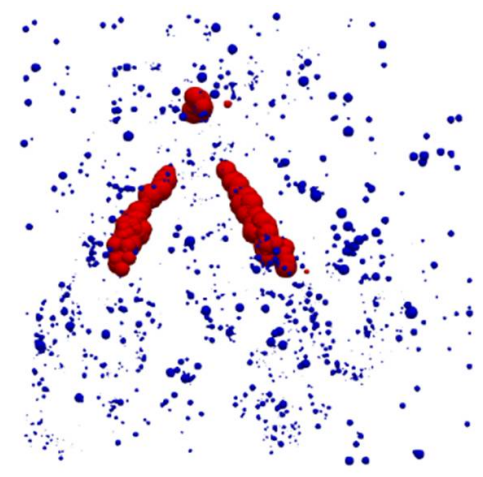

(a)

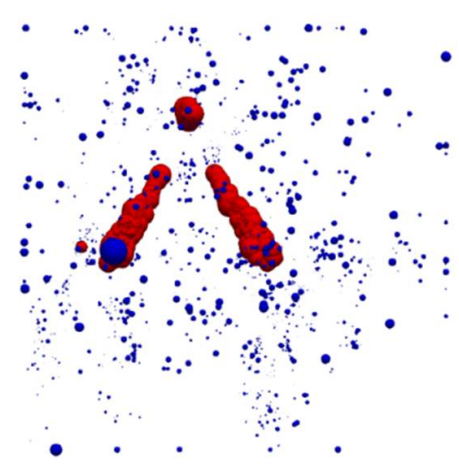

(b)

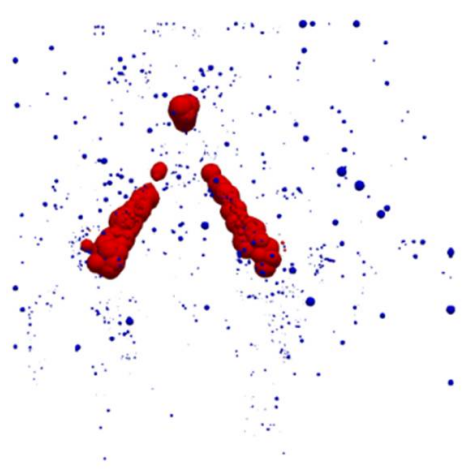

(c)

FIGURE 8. Parcels representation at 0.18 s for different velocities: a) $3 \mathrm{~m} / \mathrm{s}$, b) $6 \mathrm{~m} / \mathrm{s}$ and c) $10 \mathrm{~m} / \mathrm{s}$. Parcels upstream the POCS are coloured in red, parcels downstream are coloured in blue. Same scale factor is applied to red and blue according to the parcel diameter.

In Fig. 9 it is possible to notice that the vapour concentration at the outlet tends to spread across the outlet section, despite the distribution is less uniform with respect to what obseved in case of the liquid droplets. Moreover the average concentration decreases witht the velocity as already discussed.

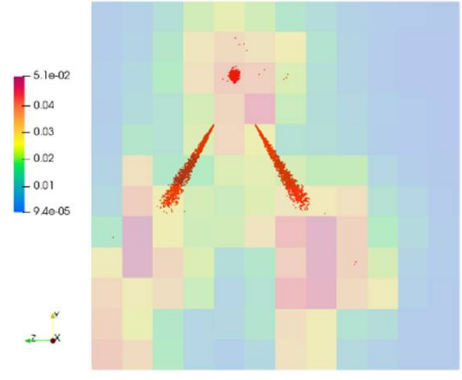

(a)

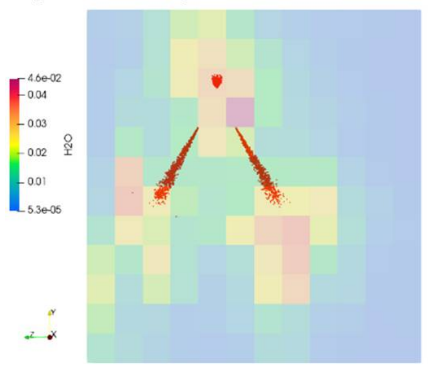

(b)

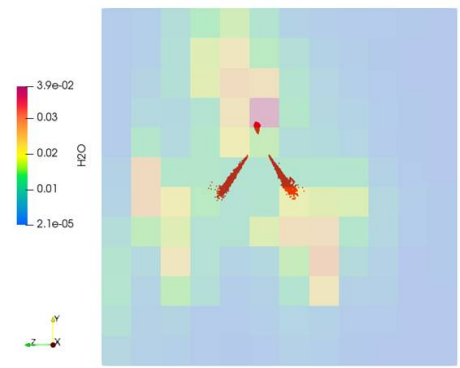

(c)

FIGURE 9. Water concentration at the outlet patch at $0.18 \mathrm{~s}$ for different velocities: a) $3 \mathrm{~m} / \mathrm{s}$, b) $6 \mathrm{~m} / \mathrm{s}$ and c) $10 \mathrm{~m} / \mathrm{s}$. Parcels upstream the POCS are superimposed.

Finally, in Fig. 10 the lateral view of the spray cloud is reported, showing the process of breakup and distribution across the POCS. This visually confirms the good performances of the POCS in enhancing the uniformity of the spray cloud distribution and suggests that the thermal breakup is the main driving force of the process. On the other hand, in order to guarantee the uniformity of the water vapour a longer substrate seems to be needed.

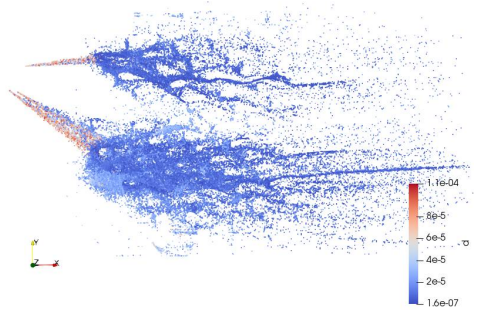

(a)

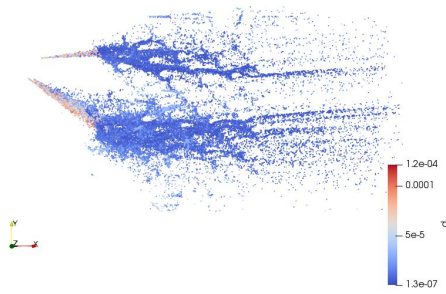

(b)

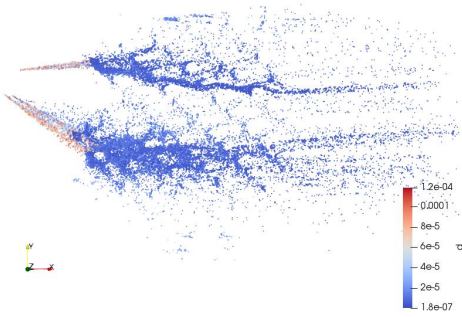

(c)

FIGURE 10. Lateral view of the water spray evolution across the POCS at $0.18 \mathrm{~s}$ for different velocities: a) $3 \mathrm{~m} / \mathrm{s}, \mathrm{b}$ ) $6 \mathrm{~m} / \mathrm{s}$ and c) $10 \mathrm{~m} / \mathrm{s}$. 


\subsection{Effects of the POCS wall temperature}

The mixing performances of the POCS have been evaluated also under variation of its wall temperature, in order to confirm that heating the structure could guarantee an higher evaporation and homogenization of the water vapour downstream the mixing section. Therefore, different temperature of the wall were considered: $300 \mathrm{~K}, 500 \mathrm{~K}$ and $800 \mathrm{~K}$. In all the cases, inlet gas temperature was kept at $500 \mathrm{~K}$ and gas velocity at $6 \mathrm{~m} / \mathrm{s}$. According to the Kuhnke model previoulsy introduced, the selected wall temperatures allows to investigate deposition/cold-splash regimes, when POCS is at $300 \mathrm{~K}\left(T^{*} \approx 0.8\right)$, and rebound/thermal breakup regimes for structure temperatures $>500 \mathrm{~K}\left(T^{*}>1.34\right)$. In the first case, evaporation will regard both film and parcels, while at the higher temperatures film formation is not possible and the evaporation is all originated from the particles.

In Fig. 11 the mass flow computed over the outlet patch for different temperatures is compared to the mass flow of the injected spray: it is worth noticing the important impact that the temperature of the POCS has on the evaporation, in particular by comparison between the curves at $500 \mathrm{~K} / 800 \mathrm{~K}$ and the $300 \mathrm{~K}$ one. In this case, the difference between the corresponding vapour mass flows is around one order of magnitude, suggesting that the mechanism of the thermal breakup has a primary role in the process.

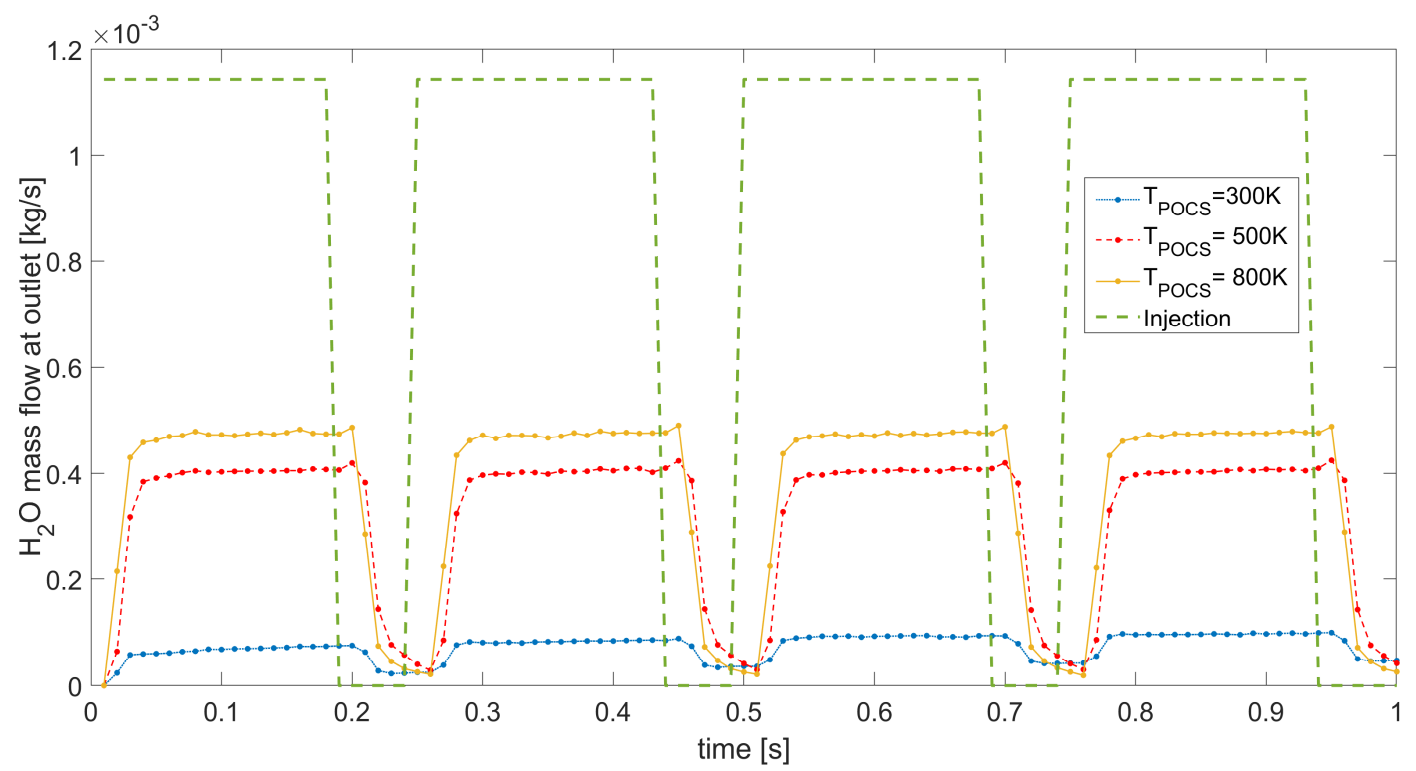

FIGURE 11. Characterization of the POCS behavior under different surface temperatures: mass flow of the evaporated water through the outlet patch.

Furthermore, the evaporated mass has been estimated by integrating the curves in Fig. 11 with respect to time and has been plotted in Fig. 12. The evaporated mass reaches values around the $40 \%$ of the total injected mass at high temperature. To be noticed that at $300 \mathrm{~K}$, as expected, a consistent part of the injected mass is stored into liquid film onto the surfaces of the POCS and, when injection is off, it partially evaporates.

In Fig. 13 the average concentration at the outlet patch has been plotted with respect to time: also in this case the effect of the temperature of the POCS guarantees an average concentration which is significantly higher for high wall temperatures. 


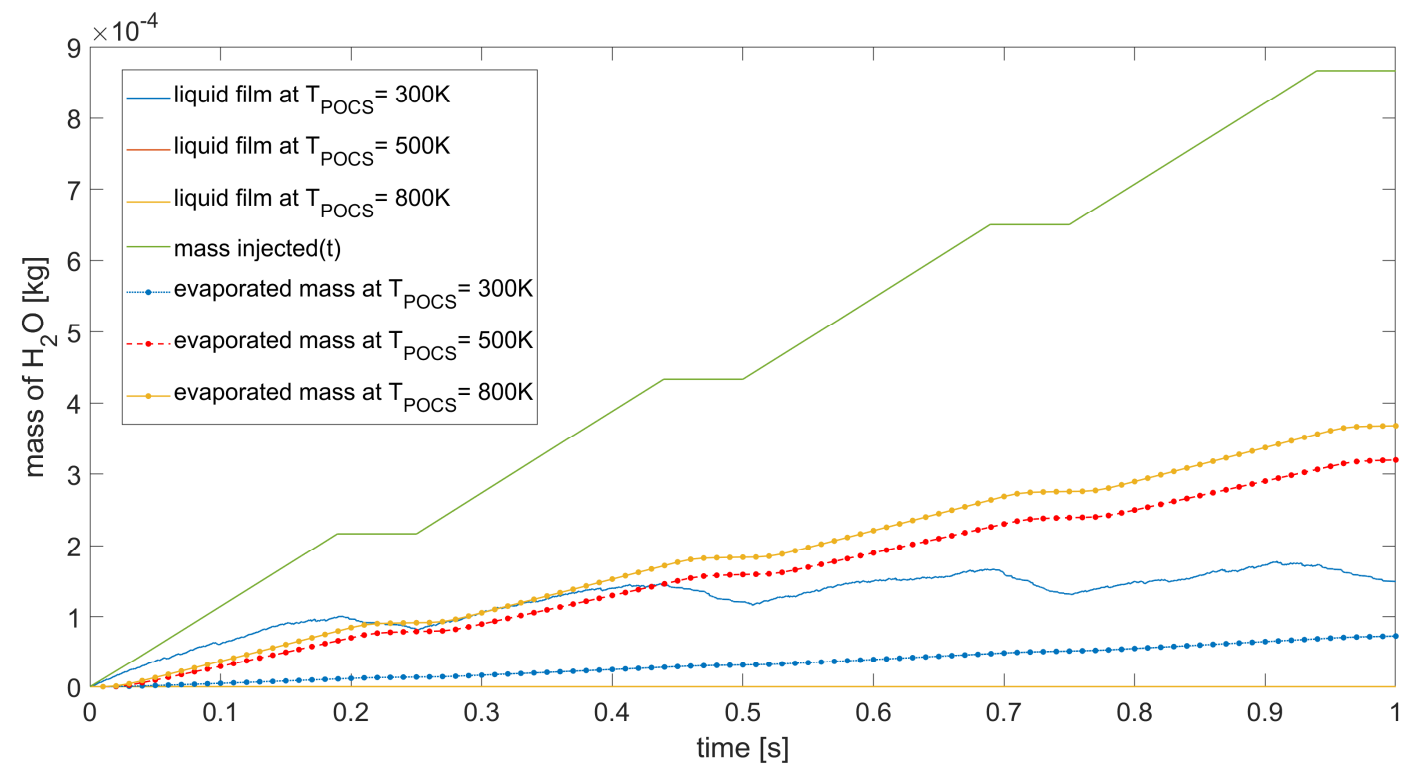

FIGURE 12. Characterization of the POCS behavior under different surface temperatures: injected liquid water mass at the inlet and evaporated water mass at the oultet.

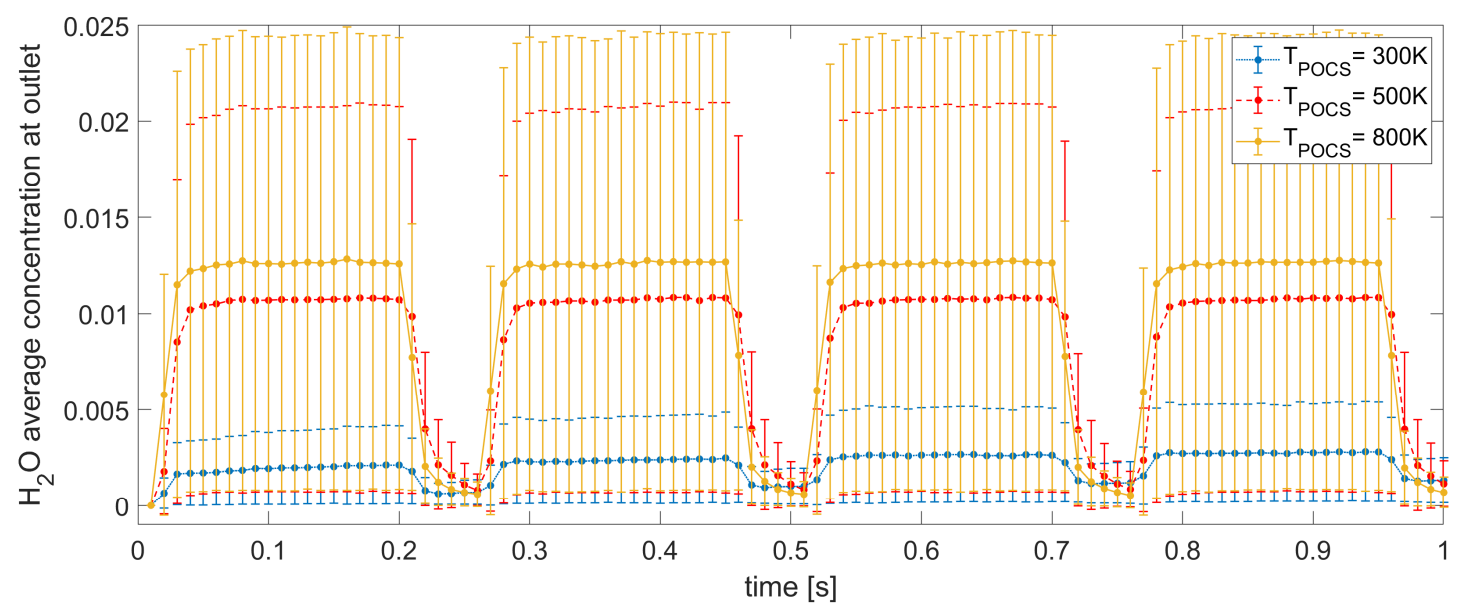

FIGURE 13. Characterization of the POCS behavior under different surface temperatures: water average concentration at the outlet patch.

In Figs. 14-16 the evolution of the spray across the POCS is shown considering the time $\mathrm{t}=0.18 \mathrm{~s}$, corresponding to the final part of the first injection. It can be distinguished a different behavior between high temperatures $(\mathrm{T}=500 \mathrm{~K}-800 \mathrm{~K})$ and low temperaure $(\mathrm{T}=300 \mathrm{~K})$. In particular, at high temperature, the thermal breakup is predominant resulting in an effective spreading of the spray cloud over the cross section of the catalyst, as described in the previous section. On the contrary, at low temperature, the process is dominated by the cold splash, resulting in a significant formation of wall film which then evaporates due to the heat exchange with the hot gas. Moreover, in this particular configuration, liquid spray is deposited on the lateral walls, leading to an accumulation of liquid phase inside the domain, which penalized the effectiveness of the evaporation and mixing process. 


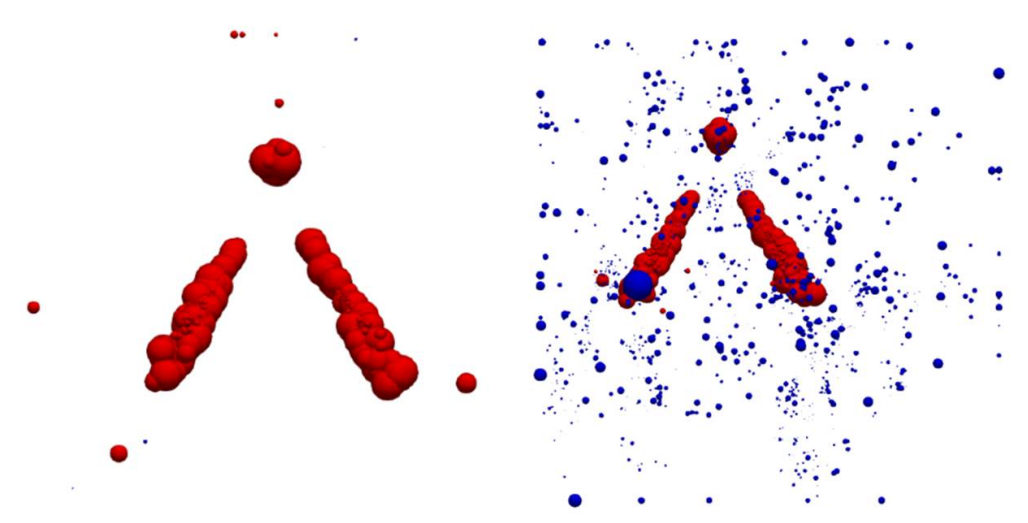

(a) (b)

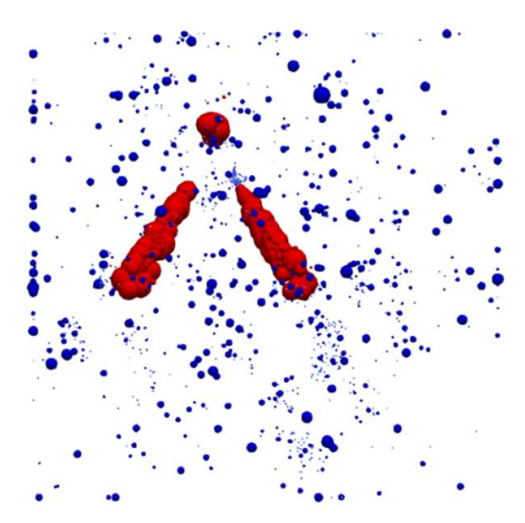

(c)

FIGURE 14. Parcels representation at $0.18 \mathrm{~s}$ for different POCS wall temperature: a) $300 \mathrm{~K}$, b) $500 \mathrm{~K}$ and c) $800 \mathrm{~K}$. Parcels upstream the POCS are coloured in red, parcels downstream are coloured in blue. Same scale factor is applied to red and blue according to the parcel diameter.

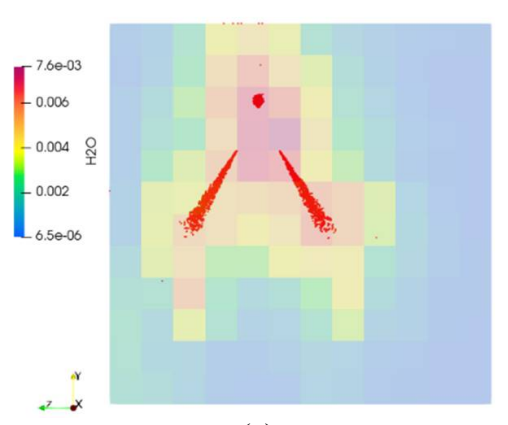

(a)

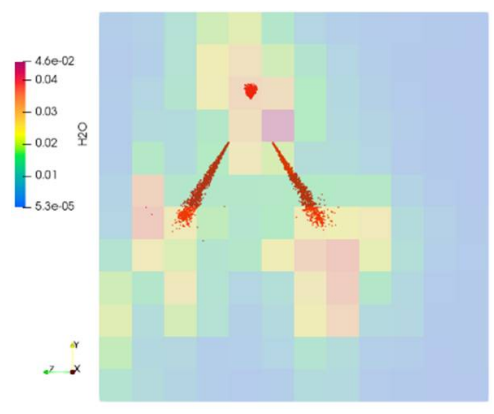

(b)

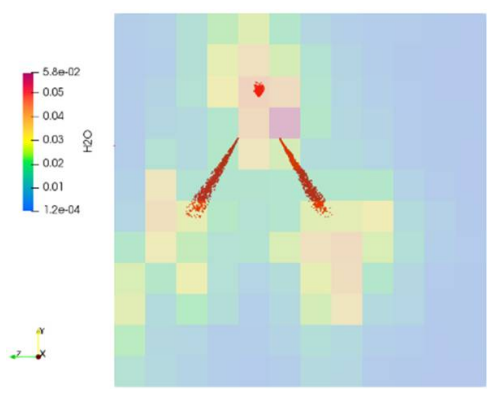

(c)

FIGURE 15. Water concentration at the outlet patch at 0.18 s for different POCS wall temperature: a) $300 \mathrm{~K}$, b) $500 \mathrm{~K}$ and c) $800 \mathrm{~K}$. Parcels upstream the POCS are superimposed.

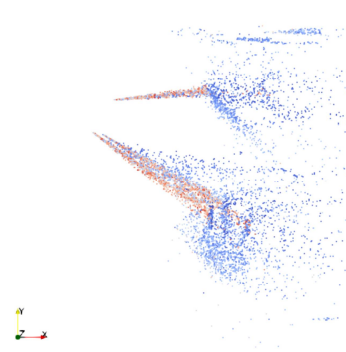

(a)

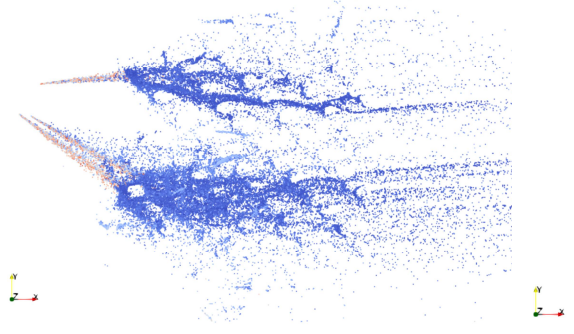

(b)

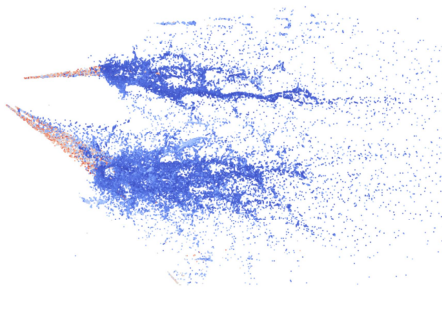

(c)

FIGURE 16. Lateral view of the water spray evolution across the POCS at 0.18 s for different POCS wall temperature: a) 300 $\mathrm{K}$, b) $500 \mathrm{~K}$ and c) $800 \mathrm{~K}$. 


\section{CONCLUSIONS}

In this paper a preliminary study on the possible application of a POCS to design a novel mixing device to be adopted in SCR systems has been presented. The numerical investigation has been carried on considering a test configuration representative of a typical SCR system in terms of gas flow velocity and temperature, cross section of the substrate, specifications of the injector and injection law. On this basis, a POCS substrate has been designed choosing a simple cubic cell, rotated by 45 degrees, as periodic elementary structure at the micro-scale. The CFD simulations have been run considering different operating conditions. The main findings of this study are hereafter summarized:

- the POCS exhibits promising performances in terms of spray-gas mixing especially at high velocities and temperature of the wall;

- the most effective mechanism for the enhancement of the spray parcels distribution is represented by the thermal breakup, which leads to the formation of small parcels which are spread across the cross-section and then evaporate in contact with the hot gas;

- the isotropic structure of the POCS allows to combine the mixer functionality with those of the catalytic substrate, since the mixing and evaporation process can proceed at the same time as the catalytic reaction occurring on the surface, if properly coated.

\section{ACKNOWLEDGMENTS}

The authors gratefully acknowledge financial support from the Ministry of Education, Universities and Research (MIUR) within the project "Department of Excellence 2018-2022: Energy for Motion".

Moreover, the valuable support of Ing. Antonello Nappi (Politecnico di Milano) during the simulation campaign is gratefully acknowledged.

\section{REFERENCES}

[1] J. Banhart, «Manufacture, characterisation and application of cellular metals and metal foams,» Progress in Materials Science, vol. 46, n. 6, pp. 559-632 (2001).

[2] L. Giani, G. Groppi e E. Tronconi, «Mass-Transfer Characterization of Metallic Foams as Supports for Structured Catalysts,» Industrial \& Engineering Chemistry Research (2002).

[3] F. Lucci, A. Della Torre, G. Montenegro e P. Dimopoulos Eggenschwiler, «On the catalytic performance of open cell structures versus honeycombs,» Chemical Engineering Journal, vol. 264, pp. 514-521 (2015).

[4] C. Bach e P. Dimopoulos Eggenschwiler, «Ceramic Foam Catalyst Substrates for Diesel Oxidation Catalysts: Pollutant Conversion and Operational Issues,» in 10th International Conference on Engines \& Vehicles, SAE International (2011).

[5] V. Papetti, P. Dimopoulos Eggenschwiler, A. Della Torre, F. Lucci, A. Ortona e G. Montenegro, «Additive Manufactured open cell polyhedral structures as substrates for automotive catalysts,» International Journal of Heat and Mass Transfer, vol. 126, pp. 1035-1047 (2018).

[6] O. Al-Ketan, M. Pelanconi, A. Ortona e R. Abu Al-Rub, «Additive manufacturing of architected catalytic ceramic substrates based on triply periodic minimal surfaces,» Journal of the American Ceramic Society, vol. 102, pp. 61766193 (2019).

[7] A. Della Torre, G. Montenegro, A. Onorati, T. Cerri, E. Tronconi e I. Nova, «Numerical Optimization of a SCR System Based on the Injection of Pure Gaseous Ammonia for the NOx Reduction in Light-Duty Diesel Engines,» in $W C X S A E$ World Congress Experience - SAE International, Detroit (2020).

[8] A. Nappi, G. Montenegro, A. Onorati e A. Della Torre, «A CHT framework for the CFD analysis of the spray-wall thermal interaction in the dosing unit of SCR systems for diesel engines,» AIP Conference Proceedings (2019). 